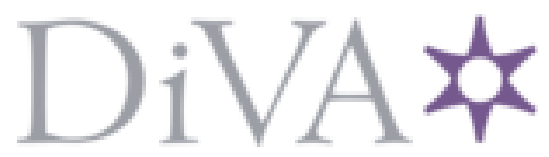

http://www.diva-portal.org

\title{
Postprint
}

This is the accepted version of a paper published in Powder Technology. This paper has been peerreviewed but does not include the final publisher proof-corrections or journal pagination.

Citation for the original published paper (version of record):

Jonsson, H., Gråsjö, J., Nordström, J., Johansson, N., Frenning, G. (2015)

An apparatus for confined triaxial testing of single particles.

Powder Technology, 270: 121-127

http://dx.doi.org/10.1016/j.powtec.2014.10.016

Access to the published version may require subscription.

N.B. When citing this work, cite the original published paper.

Permanent link to this version:

http://urn.kb.se/resolve?urn=urn:nbn:se:uu:diva-239203 


\section{An apparatus for confined triaxial testing of single particles}

Henrik Jonsson ${ }^{1}$, Johan Gråsjö ${ }^{1}$, Josefina Nordström ${ }^{1}$, Niklas Johansson ${ }^{2}$ and

Göran Frenning ${ }^{1, *}$

${ }^{1}$ Department of Pharmacy, Uppsala University, Box 580, SE-751 23 Uppsala, Sweden

${ }^{2}$ Department of Physics and Astronomy, Uppsala University, Box 516, SE-751 20 Uppsala, Sweden

*Corresponding author:

E-mail: Goran.Frenning@farmaci.uu.se, Fax: +46 18-471 42 23, Phone: +46 18-471 4375

\section{Abstract}

A novel triaxial apparatus employing overlapping rigid boundaries has been designed and constructed for experimental measurement of contact forces under confined compression of single granules in the mm-scale. The performance of the apparatus was evaluated by performing uniaxial and triaxial compression experiments on ideal elastic-plastic materials. Compression curves were compared with the fully plastic Abbott-Firestone contact model and with results from FEM simulations. The increase in contact force associated with confined conditions was observed in the compression curves from triaxial compression experiments, as supported by predictions from simulations using single particle contact models. Hence, a new method for the assessment of mechanical behaviour of single particles under confined compression can be considered as established.

Keywords: Compression, Triaxial, Single particles, Confined conditions, Apparatus design, Contact mechanics 


\section{Introduction}

Compression of granular materials is a central process within several industries, including metallurgy, pharmaceutics and ceramics $[1,2]$. To fully understand the factors influencing the performance of this process, it is necessary to assess the process characteristics on all levels, including the particle, powder and compact scales and the principles that connect them. Our main focus here is to study the mechanical behaviour at the particle level, by sorting out and experimentally study the behaviour of single particles under confined conditions by triaxial compression using a novel device designed for this purpose.

There is a multitude of devices available for the experimental characterisation of the mechanical response of single particles. An example is the classical indentation equipment where characterisation of the material and its behaviour under mechanical loading is performed by pressing a small indenter into the particle surface [3]. On a larger scale, the mechanical response of single particles is commonly assessed by using a texture analyser to uniaxially compress the particle of interest [4]. On a small scale, Atomic Force Microscopy (AFM) is a common method for measuring mechanical characteristics, either employing a cantilever with a microfabricated tip running over the sample or by using the colloidal probe technique [5].

Several micromechanical contact force relationships have been developed using the single particle approach. The classical Hertz solution [6] as well as extensions to this (see for instance Thornton et al [7] and references therein) concerning the elastic contact between spheres is one example. Another example is the similarity solution suggested by Storåkers et al. [8] for plastic deformation from particle-particle contacts. 
However, it has been known for long (see for example the work by Fischmeister and Arzt [9]) that the forces present in the powder bed in a conventional compaction process cannot be described simply as a sum of contact forces arising from an assembly of independently deforming elastic-plastic particles. The reasons for this are that at high degrees of deformation, the contact surfaces on the particle start to impinge and hence cease to be independent of each other. Mesarovic and Fleck [10] stated that this phenomenon causes the conventional models to become invalid at as low relative densities of the powder bed as about 0.8, whereas powder compaction processes needs to proceed up to relative densities as high as above 0.95 in order to form an end product of the desired quality.

For the mapping of confined single particle compression behaviour, the research has thus far been restrained to modelling by employing finite and discrete element methods (FEM/DEM) and/or by the formulation of analytical solutions. For example, Frenning [11] developed an analytical model for the micromechanical forces upon plastic deformation of particles that was validated using the FEM. Gonzalez and Cuitiño [12] attempted to overcome the restriction of independent contacts by the formulation of an analytical contact model for elastically deforming particles using an extension of the Hertz theory. Harthong et al. [13-15] have developed a contact model using a combined FEM/DEM analysis including Voronoi tessellations (as introduced to the area by Arzt [16]) specifically for studying high levels of particle densification under confined conditions. However, despite progress in contact model development, the dominating opinion within the research field still seems to be that new contact models for high degrees of densification are needed.

The challenge is therefore, as described above, to mimic the spatially confined situation to which an individual particle is exposed during a bulk powder compaction process, and thus 
enable the approximation of forces acting on the particle in such situations, something that is unachievable by compression experiments using conventional material testing equipment. The aim of this work is to set up and validate a novel apparatus that is designed for triaxial compression of single granular particles in the mm-scale. In the longer term, results extracted from the experiments performed with the apparatus will be used for the evaluation of micromechanical contact models as well as the development of new ones. The first step is, however, to investigate the ability of the apparatus to perform the type of compression experiments it is intended for. The paper is organised so that the general principle of compression is explained first, after which the design of the apparatus is presented. Finally, data from simple experiments performed by the apparatus are reported and discussed.

\section{General considerations}

The mechanism of compression employed by the apparatus involves overlapping rigid boundaries, following the principle shown in Figure 1. Upon operation, the boundaries are allowed to simultaneously, yet independently, slide over one another, gradually shrinking the rectangular box that they constitute. This enforces the sample under testing to undergo confined compression, since there is no possibility for the particle to compensate for the imposed contact forces by expanding spatially in any direction. An apparatus employing this mechanism of operation was first developed by Hambly [17] for triaxial compression of soil specimens. The principle has later been adopted for the same purpose by Airey and Wood under the name The Cambridge True Triaxial Apparatus [18]. Ibsen and Praastrup further developed the apparatus, constructing a device referred to as The Danish Rigid Boundary True Triaxial Apparatus [19]. As far as we know, this mechanism has not been employed before for the compression of single granular particles in the mm-scale. For this purpose, somewhat different issues must be considered. On one hand, no drainage is needed, since the 
samples intended for testing are virtually dry. On the other hand, the samples of interest (and thus the boundaries) are significantly smaller, posing higher demands on precision of the apparatus.

\section{Apparatus design and coordination of movements}

\subsection{Apparatus design overview}

The movements needed for the compression procedure are effectuated by three orthogonally positioned linear actuators with a loading capacity of $400 \mathrm{~N}$ each (M238.5PL, Physik Instrumente GmbH \& Co, Karlsruhe/Palmbach, Germany, described further in section 3.2). Three of the boundaries (hereafter referred to as punches, denominated $\mathrm{X}_{1}, \mathrm{Y}_{1}$ and $\mathrm{Z}_{1}$ ) are attached to the actuators which themselves are mounted to an outer aluminium frame, as seen in Figure 2. The three opposing punches $\left(\mathrm{X}_{2}, \mathrm{Y}_{2}, \mathrm{Z}_{2}\right)$ are attached to rigid aluminium cylinders, which themselves also are mounted to the outer metal frame. Upon operation, the linear actuators are set to move towards a defined position at a desired rate (see section 3.3). The distances the punches have moved are detected by position sensors integrated in the actuators and accordingly registered by in-house computer software designed in LabVIEW 2013 (National Instruments, Austin, TX, USA). The computer software simultaneously stores compression force data as registered by load cells mounted to all of the punches (see section 3.2).

In order to obtain the compression procedure of the particle according to Figure 1, the axial movement of a punch must impose lateral movements of two of the neighbouring punches (directions are defined by the coordinate system in Figure 2). This is accomplished by an inner coordination device (further described in section 3.3) that acts as a movement conveyor 
for the punches.

To enable the required lateral punch movements and to reduce frictional resistance, the actuators and aluminium cylinders connected to the $\mathrm{Y}_{1}, \mathrm{Z}_{1}, \mathrm{X}_{2}$ and $\mathrm{Y}_{2}$ punches are mounted to the outer metal frame via ball bearing rails (LLTHC $15 U$-T1 P3, SKF Sverige AB, Gothenburg, Sweden).

\subsection{Punch assembly}

Each of the linear actuators $\left(\mathrm{X}_{1}, \mathrm{Y}_{1}, \mathrm{Z}_{1}\right)$ is assembled with components as shown in Figure 3. The original actuator component consists of the parts designated 1-3 in the figure. The actuator (1) is mounted to the metal frame of the apparatus via a threaded part (2), which is screwed into place. The piston (3) is the axially movable component of the system, controlled from the computer software. To the piston, a bronze piece (4) for connection to the inner coordination device (Figure 4, see section 3.3) is attached. The distance between the flanges on the bronze piece is $6 \mathrm{~mm}$, in order to perfectly fit in the milled rectangular openings in the coordination device. At the end of the assembly, a steel punch is attached. The rod shaped tip of the punch is of $3.4 \mathrm{~mm}$ length and has a quadratic cross section with a side length of $2 \mathrm{~mm}$. The base of the punch is pyramid formed with an angle of 45 degrees. Just behind the punch, a load cell (ELFF-500N-T2M, Measurement Specialties, Les Clayes-sous-Bois, France) with a measuring capacity of $500 \mathrm{~N}$ is mounted (5), enabling direct measurement of the (normal) contact force.

$\mathrm{X}_{2}$ and $\mathrm{Y}_{2}$ are designed in the same manner as the actuator punches, but utilise rigid metal cylinders instead of actuators as attachments. Also, the distance between the flanges on the bronze pieces is $9 \mathrm{~mm}$ on these punches, thus allowing $3 \mathrm{~mm}$ of axial sliding (see section 3.3). $\mathrm{Z}_{2}$ is not connected to the inner coordination device and is, therefore, assembled without 
bronze piece, but otherwise shares the same design as $\mathrm{X}_{2}$ and $\mathrm{Y}_{2}$.

\subsection{Axial movement and spatial coordination}

The inner coordination device shown in Figure 4 is designed to coordinate the punches spatially, both when in original positions and during movement. The coordination device consists of a metal piece that has been machined by milling of five rectangular openings. Five of the six punches are mounted through sliding a bronze piece into each one of these (see Figure 4). The position of the $\mathrm{Z}_{2}$ punch is at the bottom opening of the coordination device. It is not indicated in the figure, since it is unaffected by the coordination device. The cut opening at the front of the coordination device between the $\mathrm{X}_{2}$ and the $\mathrm{Y}_{2}$ positions is machined to allow for loading of the sample.

Any actuator movement along the $\vec{x}$ or $\vec{y}$ axis is conveyed to the entire coordination device, which slides over the bronze piece mounted to the opposing punch, thus bringing the boundaries towards each other, as can be seen in Figure 5 . Along the $\vec{z}$ axis, only $\mathrm{Z}_{1}$ is attached to the coordination device, as mentioned earlier, and $\mathrm{Z}_{1}$ hence follows the same mechanism of movement as $\mathrm{X}_{1}$ and $\mathrm{Y}_{1} . \mathrm{Z}_{2}$ is completely fixed to the outer frame and is thus not affected by any movements of the other punches. A summary of how the axial movement of a punch is conveyed to the other punches is provided in Table 1.

\section{Apparatus performance testing}

\subsection{Materials}

The ability of the apparatus to compress materials under confined conditions and to accurately measure the mechanical response was tested using nominally spherical cellulose acetate (CA) and lead samples. These are known to be approximately ideal elastic-plastic materials and are 
therefore suitable for probing mechanical deformation. In order to be able to compensate for inherent (elastic) system deformations when compressing materials, uniaxial and triaxial system deformation probing was performed. The deformation was calculated from triaxial experiments with nominally inert (in terms of mechanical deformation at the applied amounts of stress) copper cubes and from uniaxial punch-to-punch experiments.

Spherical CA particles with a diameter of $2.0 \mathrm{~mm}$ were purchased from Engineering Laboratories Inc. (Oakland, NJ, USA). Nominally spherical lead particles ("Gyttorp Lead Max”) with a diameter of $2.0 \mathrm{~mm}$ were obtained from Gyttorp (Ingelstad, Sweden). Copper cubes with the side length $1.8 \mathrm{~mm}$ were manufactured by Ångströmverkstad (Uppsala, Sweden).

\subsection{Punch interference assessment}

All of the punches were aligned in positions $2 \mathrm{~mm}$ from punch-to-punch contact without any sample loaded into the device. From this position, all axially mobile punches were set to move $0.8 \mathrm{~mm}$ inwards, all at the same rate. The experiment was repeated 10 times.

\subsection{Analysis of inherent system deformations}

\subsubsection{Uniaxial probing}

One of the opposing pairs of punches was arranged at punch-to-punch contact, from which the axially mobile punch was set to move $0.08 \mathrm{~mm}$ inwards. The two remaining pairs of punches were held in constant positions where they did not interfere with the moving punches. This experiment was repeated 10 times for each opposing pair of punches.

\subsubsection{Triaxial probing}

A copper cube was loaded into the device, after which all of the punches were moved inwards 
manually until they registered a similar contact force value (about $20 \mathrm{~N}$ ) on all load cells. From there, the axially mobile punches were set to move $0.08 \mathrm{~mm}$ inwards at the same rate. The experiment was repeated 10 times.

\subsection{Particle compression experiments}

\subsubsection{Uniaxial particle compression}

CA and lead particles were loaded into the apparatus, one at a time, after which one of the axially mobile punches was set to move inwards at a rate of $10 \mu \mathrm{m} / \mathrm{s}$ pressing the particle against the opposing punch. The remaining punches were drawn aside and held in positions where they did not interfere with the moving punches or the particle. This experiment was repeated 10 times for each particle type and each opposing pair of punches.

\subsubsection{Triaxial particle compression}

CA and lead particles were loaded, one at a time, into the apparatus so that the particle and the punches precisely made contact. From there, all of the axially mobile punches were set to move simultaneously inwards at a rate of $10 \mu \mathrm{m} / \mathrm{s}$. This experiment was repeated 10 times for each particle type.

\subsubsection{Comparison with contact models}

A FEM model developed and described by Frenning [11] for confined compression of single particles was used for comparison with the experimental results from the compression of CA particles. For this simulation, parameters corresponding to spherical particles with a diameter of $2 \mathrm{~mm}$ were used. According to literature [20], the yield strength $\left(\sigma_{Y}\right)$ was set to $37 \mathrm{MPa}$ and the elastic modulus $(E)$ to $20 \mathrm{GPa}$. A Poisson ratio (v) of 0.3 was used. No simulations were performed for the lead particles, since their less ideal shape would render a comparison between experiments and simulations inconclusive. 
The experimental results for both the particle types were also compared with the theoretical fully plastic Abbott-Firestone (AF) contact model (described in detail by Jackson and Green [21]). This model assumes that the normal contact force between a (hemi)sphere and a rigid flat surface can be described as a linear function of the material hardness (assumed to be constant), the initial radius of the sphere and the degree of compression of the sphere at any given instance:

$$
F_{c}=2 \pi R H \omega
$$

Here, $F_{c}$ is the contact force, $H$ is the hardness, $R$ is the initial radius and $\omega$ is the particle overlap. The hardness has in turn been shown to be accurately described as a multiple of the yield strength of the material. Jackson and Green described the hardness as:

$$
H=2.8 \sigma_{Y}
$$

where $\sigma_{Y}$ is the yield strength of the material. For lead and CA, the yield strength values used were $20 \mathrm{MPa}$ (from [22]) and $37 \mathrm{MPa}$ (from [20]), respectively.

\section{Results and discussion}

In all of the results presented here, only the load cells mounted to the $X_{2}, Y_{2}$ and $Z_{2}$ punches were accounted for, whereas the load cells on the actuators were only used as references during the experiments.

\subsection{Punch interference assessment}


As can be seen from the experimental data in Figure 6, no interferences from the system were observed on the $\mathrm{X}_{2}$ and $\mathrm{Y}_{2}$ load cells, whereas some stray forces (around $10 \mathrm{~N}$ at maximum) were registered by $Z_{2}$. Since these forces are relatively small and uniform, the discrepancy from actual forces will be practically negligible and the registered forces during particle compression experiments will therefore be considered as accurate.

\subsection{Theoretical inherent system deformation}

\subsubsection{Uniaxial}

Typical curves from the uniaxial system deformation probing are shown in Figure 7. The curves can all be considered as linear $\left(R^{2} \geq 0.9995\right)$, but with somewhat different slopes. The deformation per force unit registered by $\mathrm{X}_{2}$ and $\mathrm{Y}_{2}$ can be regarded as similar $(0.397 \pm 0.002$ $\mu \mathrm{m} / \mathrm{N}$ and $0.386 \pm 0.016 \mu \mathrm{m} / \mathrm{N}$, respectively) whereas $\mathrm{Z}_{2}(0.262 \pm 0.004 \mu \mathrm{m} / \mathrm{N})$ showed a smaller deformation per force unit than the others. These discrepancies can be explained by considering that the deformation may be caused not solely by the punches. There is, for example, complex force transmission via the inner coordination device as well as gravitational forces and leverage from the actuators and deformations in the outer frame.

\subsubsection{Triaxial}

The triaxial system deformation probing showed similar curves for all the punches at small deformations, whereas some deviations from the initial linearity were observed at larger deformations $\left(R^{2} \geq 0.9957\right)$. However, these discrepancies tended to undulate around an average, and a value of $0.363( \pm 0.065) \mu \mathrm{m} / \mathrm{N}$, corresponding to a mean from the all of the triaxial system deformation probing experiments, was hence used to compensate for system deformations in the triaxial compression experiments throughout the study.

It can be noted from the typical probing curves in Figure 8 that the $\mathrm{Z}_{2}$ curve clearly does not 
show the same extent of deviation from the $\mathrm{X}_{2}$ and $\mathrm{Y}_{2}$ curves as in the uniaxial case. Hence, the mechanism with which forces distribute throughout the system is different when all of the punches are involved in the compression instead of only one opposing pair of punches. The uniform distribution of forces in the triaxial case is rather expected for a symmetric system such as the one constructed here, and hence lends support to the hypothesis of complex force transmission paths when compressing uniaxially, as presented in section 5.2.1.

\subsection{Apparatus performance under triaxial compression}

Typical compression curves from triaxial experiments as well as curves presenting average values and standard deviations on CA and lead are shown in Figures $9 a$ and $9 b$. It can be noted that all of the load cells registered similar curve shapes and approximately the same contact force value at the corresponding strain level during a run for both the materials, which also should be the case if the apparatus operates correctly.

From run to run, the curves from compression of CA particles (Figure 9c) showed high uniformity and hence a high degree of reproducibility. The contact forces registered from compression of lead particles, on the other hand, showed fluctuations between runs, as seen in Figure 9d. Even though a strict increase of average stress with strain was observed up to an engineering strain $\varepsilon=0.2$ for the lead particles, the uncertainties observed cannot be neglected. However, these fluctuations could be explained by the fact that the CA particles are engineered to be perfectly spherical and monosized, whereas the lead particles are made with the ambition only to be nominally spherical and with less precision in particle diameter. These shape-variations also caused the load cells at the different punches (which ones differed from run to run) to register the first contact with the lead sample at different levels of strain, which was not the case for the CA particles. 
Due to the existence (or the arising) of gaps between the punch boundaries during compression, the particles had a tendency to extend outside the rectangular cavity at a certain point during the compression and hence depart from the confined conditions. This can be seen at $\varepsilon=0.22$ for the lead particles, where the compression curve start to bend downwards. The same tendency was also observed for CA at higher strain levels (not shown in the figure). This behaviour is not desired, but does on the other hand occur at strain levels where all the data of interest already has been collected and thus does not influence the results to a significant extent.

\subsection{The ability of the apparatus to compress under confined conditions}

The triaxial and uniaxial compression curves for both of the materials tended to follow the same trend up to a certain degree of strain (about 0.1), as can be seen in Figure 10. From that point, the triaxial compression curve showed a significantly steeper slope than the uniaxial curve. As mentioned earlier, this phenomenon is associated with the plastic incompressibility and contact impingement related to confined conditions.

The curves demonstrating the results from uniaxial and triaxial simulations showed the same shape as the corresponding curves from the experiments, within the range of interest. The triaxial compression curve also deviates from the uniaxial compression curve at approximately the same degree of strain in both experiments and simulations. At high strains, the slope of the triaxial simulation curve becomes very large, whereas the slope of the curve from triaxial compression experiments rather flattens. The reason for this most likely is the tendency of the sample to evade the confined state by extending through emerging gaps between the boundaries. The curves from experiments and simulation of uniaxial compression of CA particles follow each other very well within the range of interest. All in all, after considering these results it seems reasonable to claim that confined compression has been 
performed using the apparatus, when compressing triaxially.

The Abbott-Firestone model showed resemblance with the linear regions of the experimental results from uniaxial compression of lead particles. The different offset in contact force for the AF model and the experimental results for lead can be explained by the non-spherical particle shape as well as the viscoelastic behaviour commonly observed when compressing lead [22]. In the case of uniaxial CA particle compression, the AF model showed a satisfactory agreement both with experimental results and the simulation curves for small

strains. However, discrepancies were evident for strains exceeding about $10 \%$. The reason for this could be that the AF model describes the contact force from a single contact between a hemisphere and a rigid flat surface. However, under uniaxial particle compression, there are two such contacts acting on the same particle. Even though these contacts do not impinge, the propagation of plastic deformation tend to lead to coalescence of plastically deformed zones at higher strains (see for example [11]), thus affecting the contact forces. Compared to the triaxial curves, the AF model assumes full plasticity and contact independence and hence becomes invalid in modelling triaxial compression at strain levels where contact impingement becomes significant, which also can be seen in Figure 10.

\section{Conclusions}

An apparatus for testing of single particles in the mm-scale under confined conditions has been constructed and tested for ideal materials with satisfying results. This opens up an array of possibilities for future testing of materials on the single particle level. One may for example perform experiments involving particles of different compositions, shapes and porosities, as well as experiments imposing different stress levels along the radial and axial 
directions. Our main interests are within the area of pharmaceutical powders. However, as shown in this work, nothing precludes testing of other materials.

There are still improvements to be made in order to optimise the performance of the apparatus. The original idea was to construct the apparatus without the inner coordination device, since the punches were thought to be arrangeable in space in such a way that they could constitute the inner rectangular box without any spatial guidance at the boundary level. As we have seen, this would not be possible, since the configuration was difficult to establish and maintain even with the coordination device present. A more precise inner guiding could possibly reduce imperfections such as stray forces as well as gaps between the punches appearing upon compression.

\section{Acknowledgments}

This study was supported by a grant provided by the Swedish Research Council (No. 6212011-4049).

\section{References}

[1] Larsson PL, Biwa S, Storakers B. Analysis of cold and hot isostatic compaction of spherical particles. Acta Materialia. 1996 Sep;44(9):3655-66.

[2] Tho I, Bauer-Brandl A. Quality by design (QbD) approaches for the compression step of tableting. Expert Opinion on Drug Delivery. 2011 Dec;8(12):1631-44.

[3] Cheng L, Xia X, Scriven LE, Gerberich WW. Spherical-tip indentation of viscoelastic material. Mech Mater. 2005 Jan;37(1):213-26.

[4] Nordstrom J, Welch K, Frenning G, Alderborn G. On the physical interpretation of the Kawakita and Adams parameters derived from confined compression of granular solids. Powder Technol. 2008 Mar;182(3):424-35.

[5] Butt HJ, Cappella B, Kappl M. Force measurements with the atomic force microscope: Technique, interpretation and applications. Surf Sci Rep. 2005 Oct;59(1-6):1-152. 
[6] Hertz H. Ueber die Berührung fester elastischer Körper. Journal für die reine und angewandte Mathematik 1881;92:156-71.

[7] Thornton C, Cummins SJ, Cleary PW. An investigation of the comparative behaviour of alternative contact force models during elastic collisions. Powder Technol. $2011 \mathrm{Jul}$ 10;210(3):189-97.

[8] Storåkers B, Biwa S, Larsson PL. Similarity analysis of inelastic contact. Int J Solids Struct. 1997 Aug;34(24):3061-83.

[9] Fischmeister HF, Arzt E. Densification of powders by particle deformation. Powder Metallurgy. 1983;26:82-8.

[10] Mesarovic SD, Fleck NA. Frictionless indentation of dissimilar elastic-plastic spheres. Int J Solids Struct. 2000 Nov;37(46-47):7071-91.

[11] Frenning G. Towards a mechanistic model for the interaction between plastically deforming particles under confined conditions: A numerical and analytical analysis. Materials Letters. 2013 Feb 1;92:365-8.

[12] Gonzalez M, Cuitino AM. A nonlocal contact formulation for confined granular systems. Journal of the Mechanics and Physics of Solids. 2012 Feb;60(2):333-50.

[13] Harthong B, Jerier JF, Doremus P, Imbault D, Donze FV. Modeling of high-density compaction of granular materials by the Discrete Element Method. Int J Solids Struct. 2009 Sep;46(18-19):3357-64.

[14] Harthong B, Jerier J-F, Richefeu V, Chareyre B, Doremus P, Imbault D, et al. Contact impingement in packings of elastic-plastic spheres, application to powder compaction.

International Journal of Mechanical Sciences. 2012 Aug;61(1):32-43.

[15] Jerier JF, Hathong B, Richefeu V, Chareyre B, Imbault D, Donze FV, et al. Study of cold powder compaction by using the discrete element method. Powder Technol. 2011 Mar;208(2):537-41.

[16] Arzt E. Influence of an increasing particle coordination on the densification of spherical powders. Acta Materialia. 1982;30:1883-90.

[17] Hambly EC. A new Triaxial Apparatus. Geotechnique. 1969;19:307-9.

[18] Airey DW, Wood DM. The Cambridge True Triaxial Apparatus. Advanced Triaxial Testing of Soil and Rock 1988:796-805.

[19] Ibsen LB, Praastrup U. The Danish rigid boundary true triaxial apparatus for soil testing. Geotechnical Testing Journal. 2002 Sep;25(3):254-65.

[20] Braganca FC, Rosa DS. Thermal, mechanical and morphological analysis of poly(epsilon-caprolactone), cellulose acetate and their blends. Polym Adv Technol. 2003 Oct;14(10):669-75. 
[21] Jackson RL, Green I. A finite element study of elasto-plastic hemispherical contact against a rigid flat. J Tribol-Trans ASME. 2005 Apr;127(2):343-54.

[22] Lyons WJ. Plastic deformation of lead, copper and aluminum under static compression. J Appl Phys. 1938 Oct;9(10):641-6. 
a)

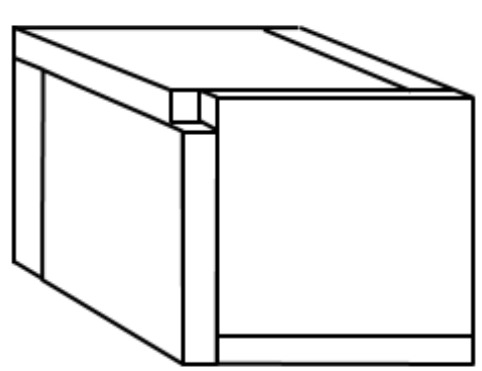

c)

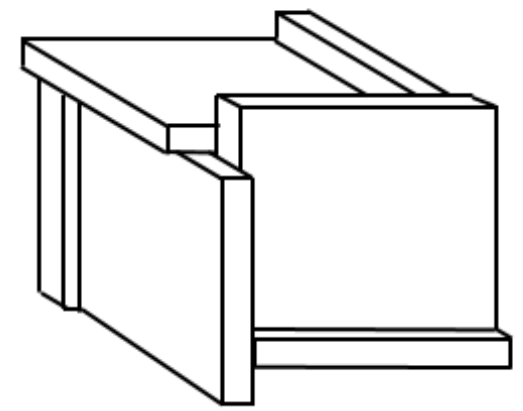

b)

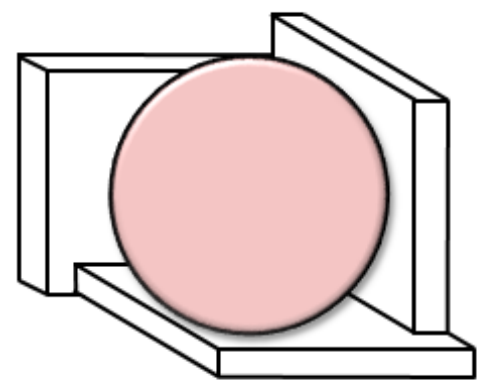

d)

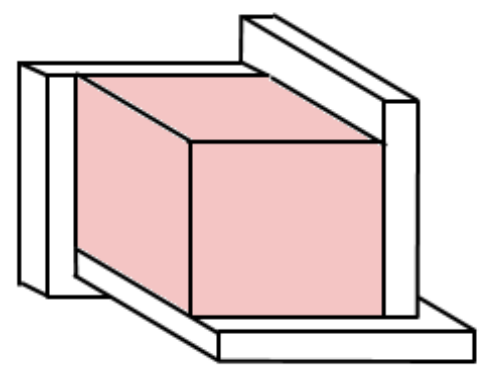

Figure 1 - The mechanism of operation of the rigid boundary triaxial apparatus. Six partially overlapping rigid boundaries form an initially cubic cavity (a) into which a particle is placed (b). During operation, the boundaries are allowed to slide past each other (c), eventually compressing the particle into a rectangular shape (d).

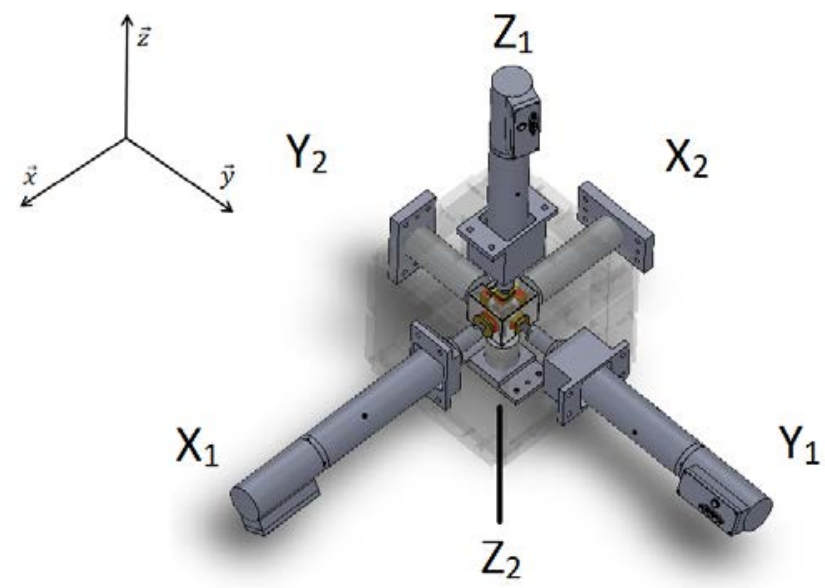

Figure 2 - Drawing of the triaxial apparatus seen obliquely from above, involving denomination of the punches. For clarity, the metal frame is shown as shaded. Also shown is the coordinate system defining the directions of punch movements. 


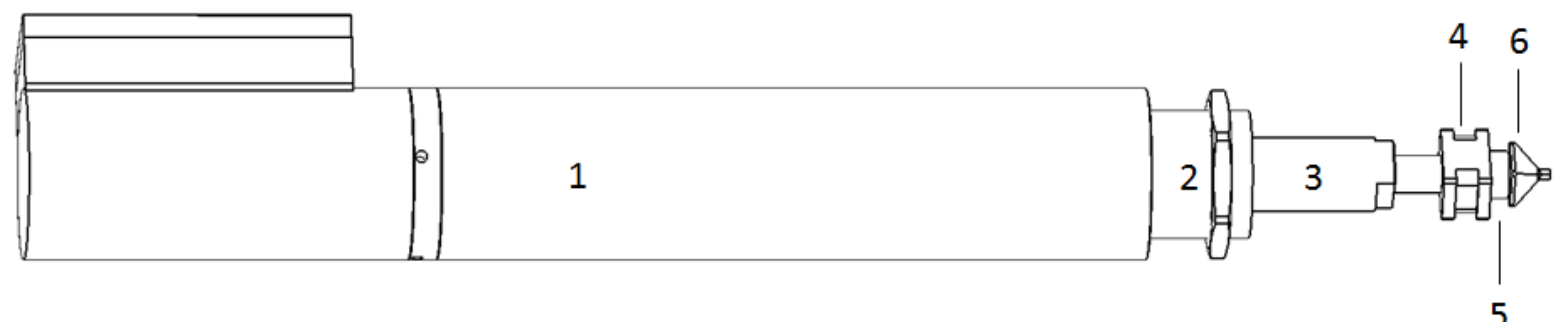

Figure 3 - Schematic of a linear actuator and its attached components: 1. Central part of the actuator. 2. Threaded part for attachment to the metal frame. 3. Axially movable piston. 4. Bronze piece for attachment to the inner coordination device. 5. Load cell. 6. Pyramidal punch.

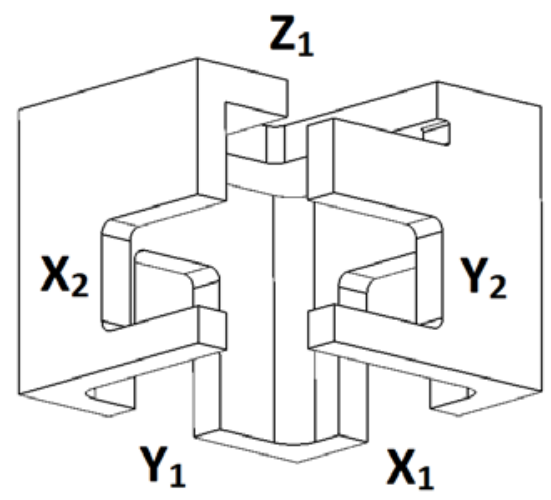

Figure 4 - Schematic of the inner coordination device. The positions of the different punches (except $\mathrm{Z}_{2}$ ) are indicated in the figure.
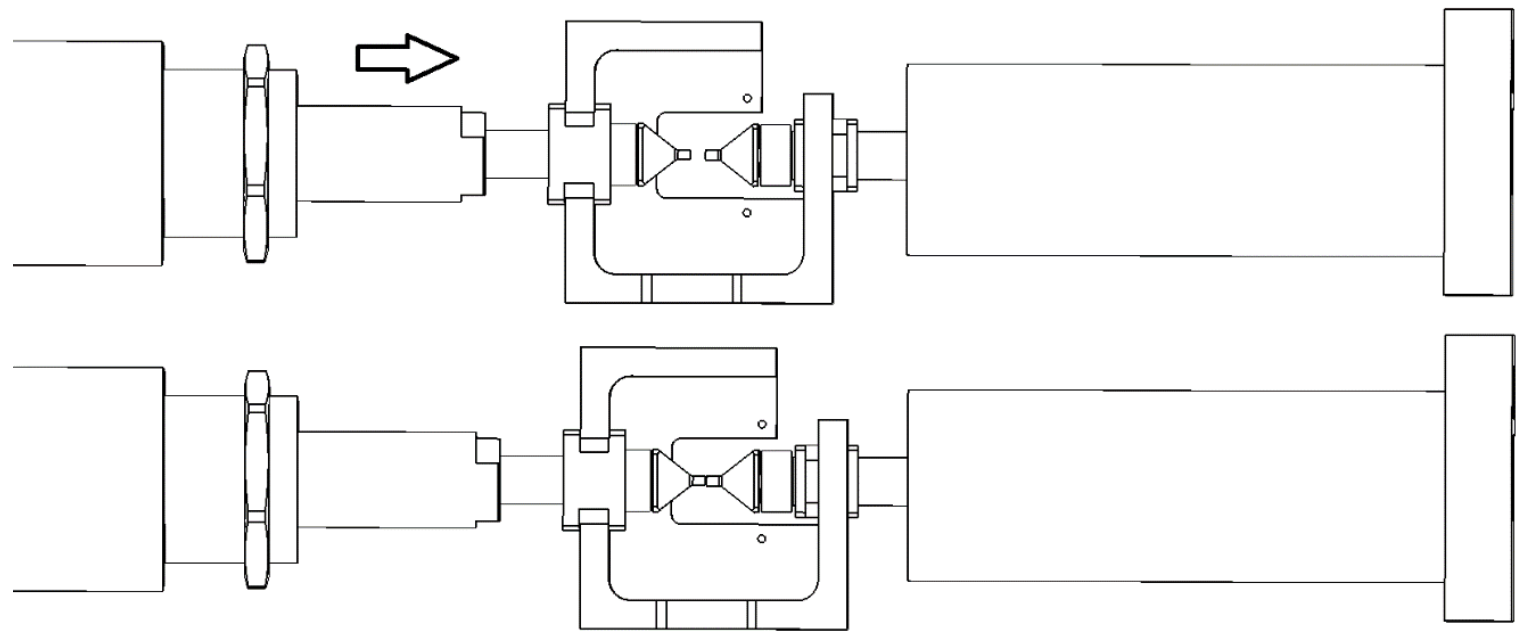

Figure $\mathbf{5}$ - Mechanism of axial movement along the $\overrightarrow{\mathbf{x}}$ and $\overrightarrow{\mathbf{y}}$ axes, viewed from below the coordination device. For clarity, only the $\mathrm{Y}_{1}$ (left) and $\mathrm{Y}_{2}$ punches are shown. 


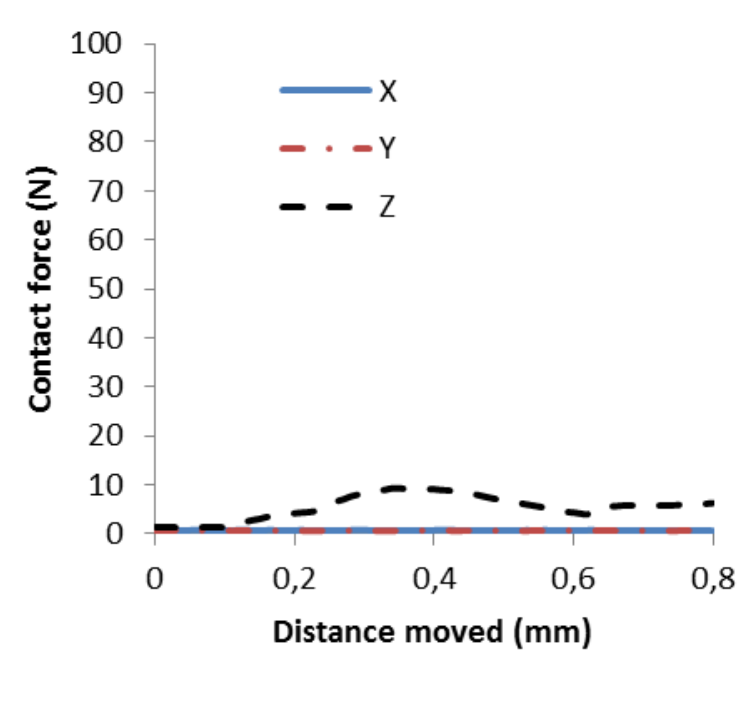

Figure 6 - Contact forces registered for triaxial punch movements without sample.

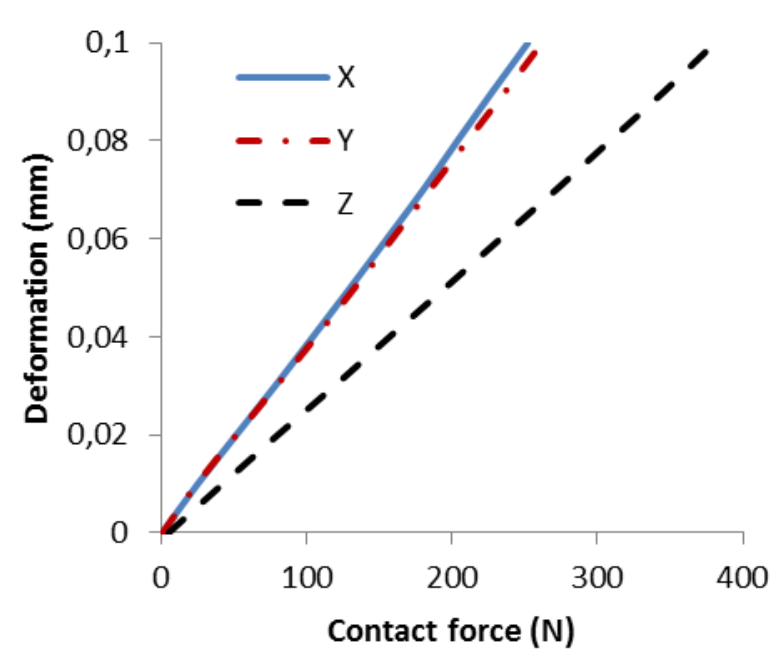

Figure 7 - Typical curves for system deformations at different contact forces during uniaxial compression. 


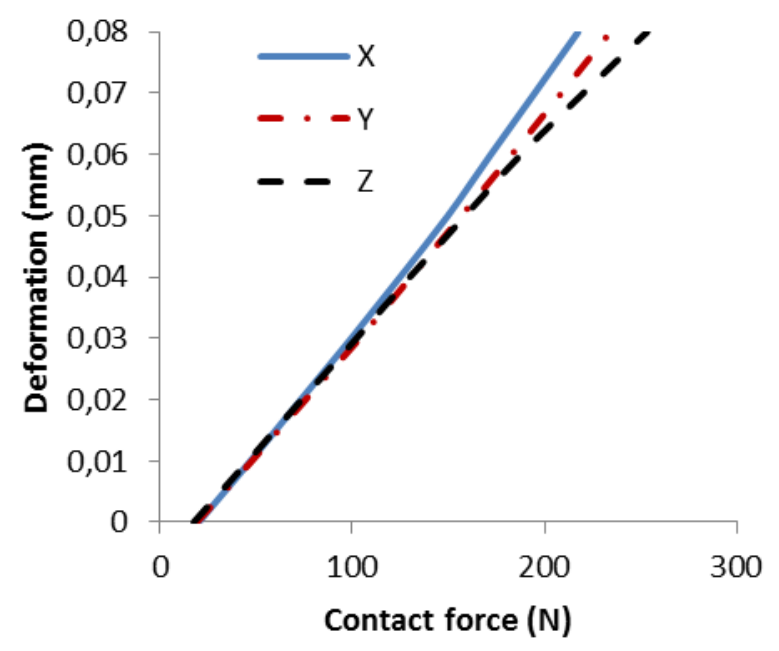

Figure 8 - Typical curves for system deformations at different contact forces during triaxial compression.

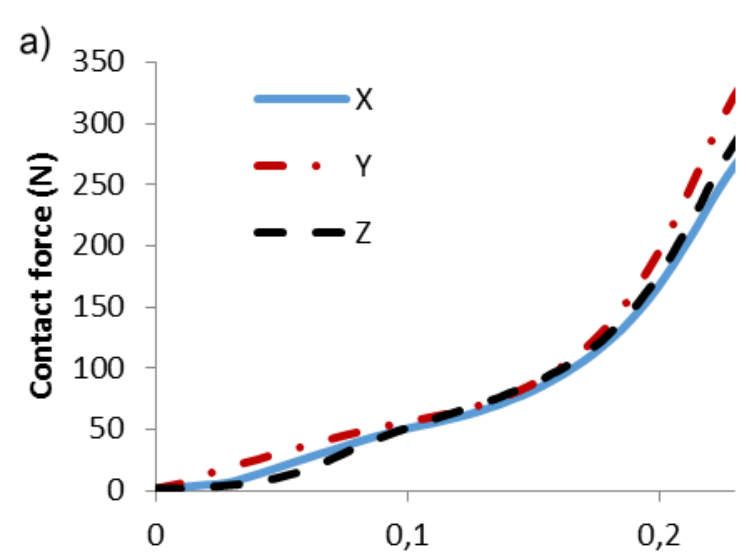

b) 350
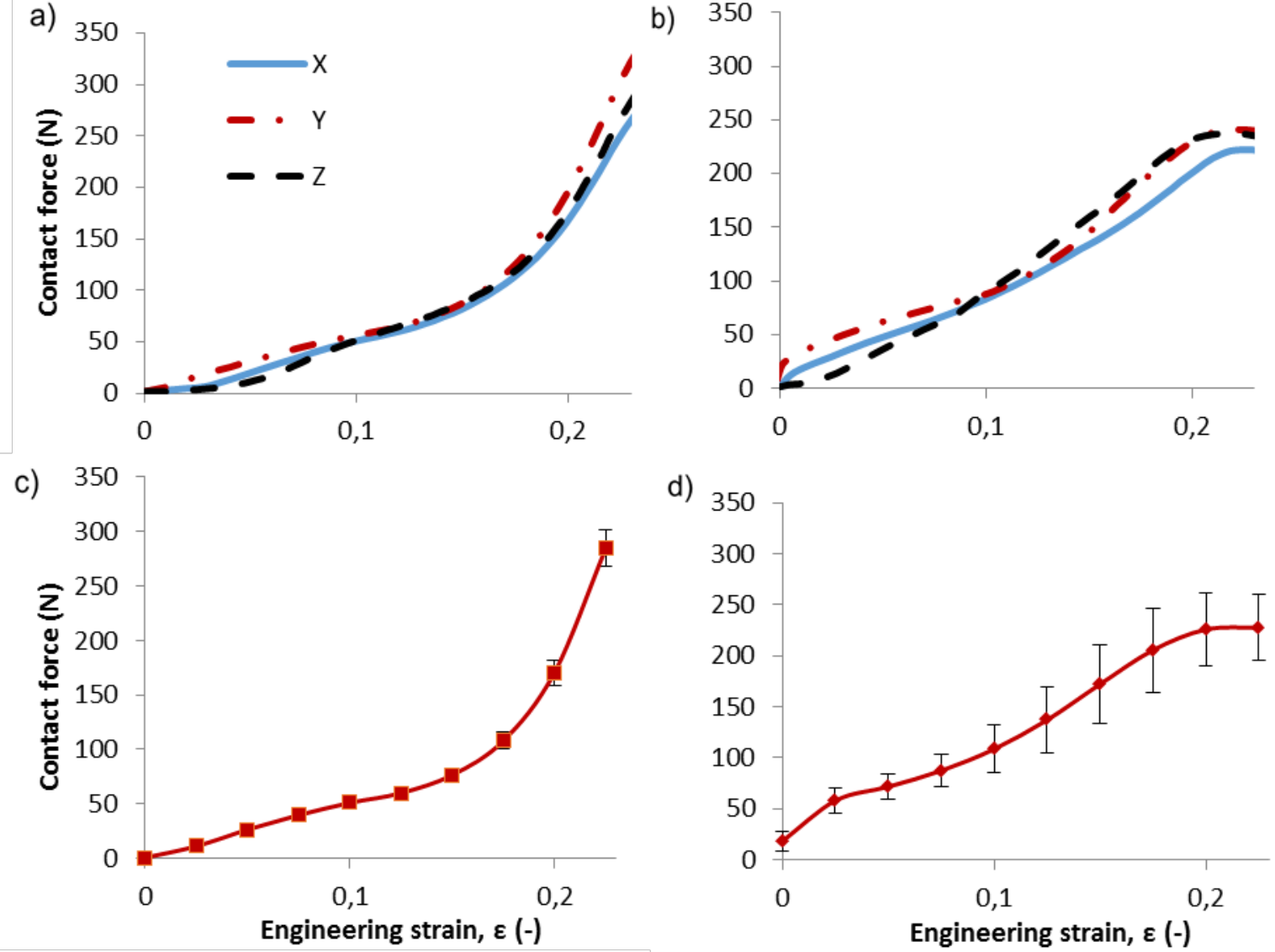

d)

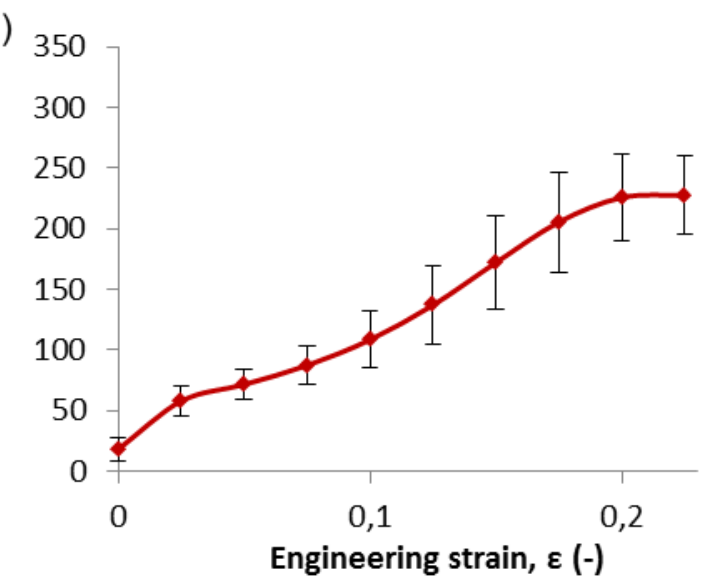

Figure 9 - Top: Typical compression curves from the triaxial compression experiments on cellulose acetate (a) and lead particles (b) measured along all axes. Bottom: Average contact pressure values and standard deviations from 10 triaxial compressions of cellulose acetate (c) and lead (d) particles at 10 different strain levels, measured by the $\mathrm{Y}_{2}$ load cell. 

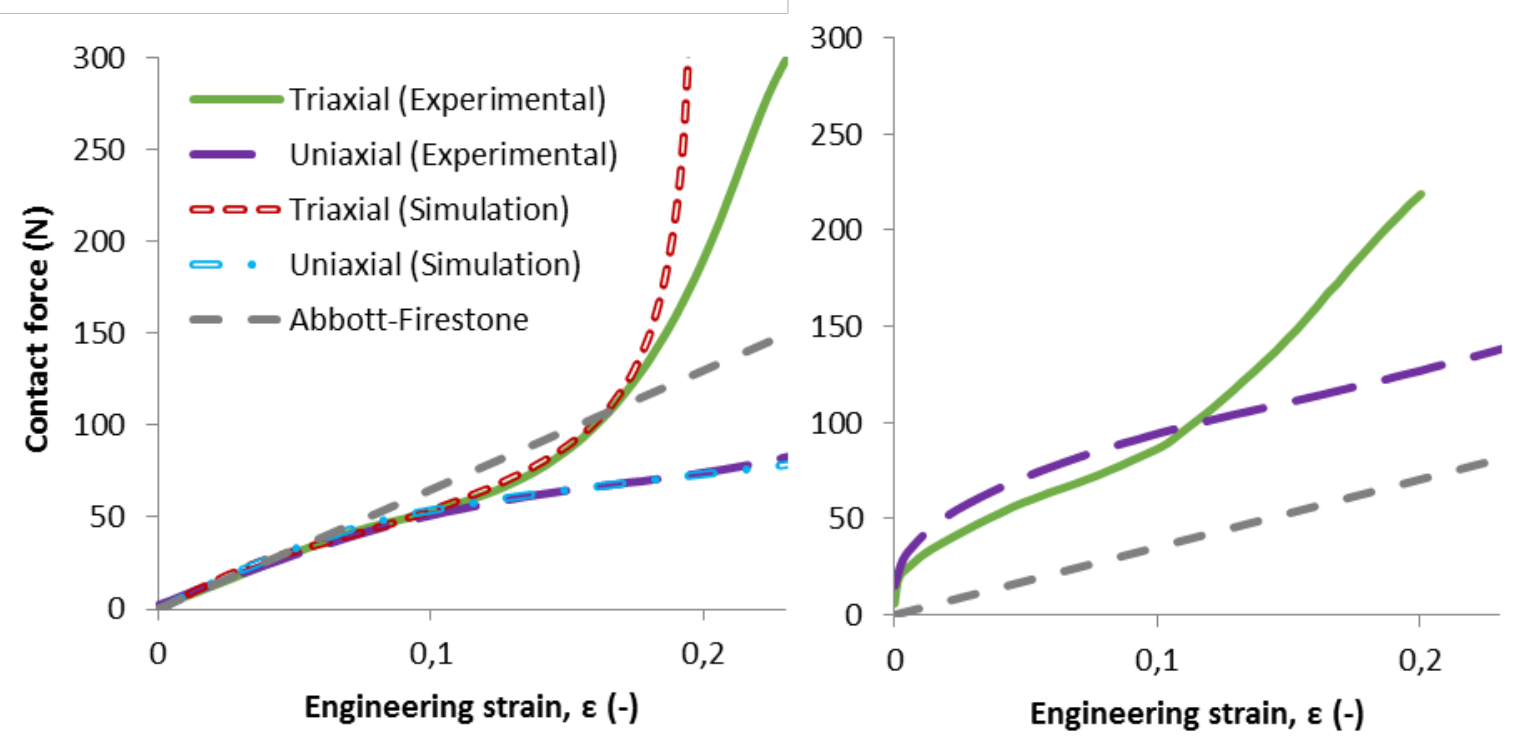

Figure 10 - Left: Comparison between the theoretical Abbott-Firestone model, finite element simulations and typical results from experiments involving triaxial and uniaxial compression of cellulose acetate particles. Right: Comparison between the Abbott-Firestone model and typical results from experiments involving triaxial and uniaxial compression of lead particles.

Table 1 - Conveying of movements between punches mediated by the coordination device.

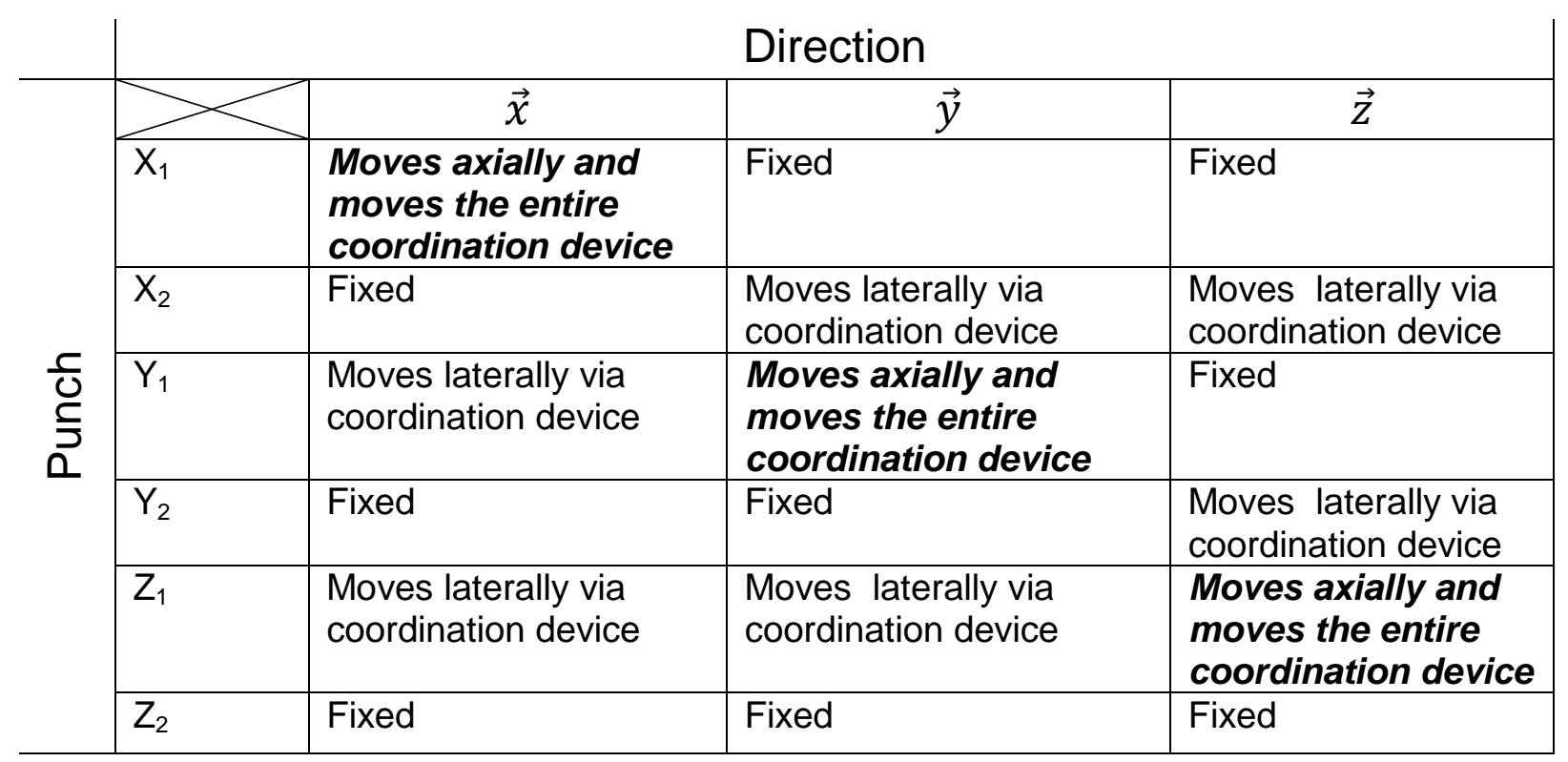

